\title{
Design, Synthesis and Biological Evaluation of Novel Pyrazole Sulfonamide Derivatives as Potential AHAS Inhibitors
}

\author{
Xian-Hai Lv, ${ }^{*, a}$ Zi-Li Ren, ${ }^{b}$ Hao Liu, ${ }^{a}$ Hai-dong Li,${ }^{c}$ Qing-Shan Li, ${ }^{* d, e}$ Li Wang, ${ }^{a}$ Li-Song Zhang, ${ }^{a}$ \\ Xiao-Kang Yao, ${ }^{a}$ and Hai-Qun $\mathrm{Cao}^{b}$ \\ ${ }^{a}$ School of Science, Anhui Agricultural University; Hefei 230036, P. R. China: ${ }^{b}$ School of Plant Protection, Anhui \\ Agricultural University; Hefei 230036, P. R. China: ${ }^{c}$ School of Materials, The University of Manchester; Oxford

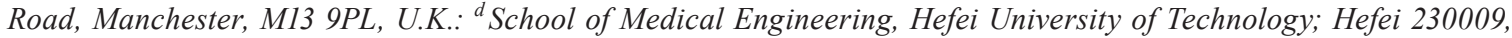 \\ P. R. China: and ${ }^{e}$ State Key Laboratory of Pharmaceutical Biotechnology, Nanjing University; Nanjing 210093, P. R. \\ China. \\ Received September 20, 2017; accepted December 28, 2017
}

Acetohydroxy acid synthase (AHAS; EC 2.2.1.6, also referred to as acetolactate synthase, ALS) has been considered as an attractive target for the design of herbicides. In this work, an optimized pyrazole sulfonamide base scaffold was designed and introduced to derive novel potential AHAS inhibitors by introducing a pyrazole ring in flucarbazone. The results of in vivo herbicidal activity evaluation indicates compound $3 \mathrm{~b}$ has the most potent activity with rape root length inhibition values of $81 \%$ at $100 \mathrm{mg} / \mathrm{L}$, and exhibited the best inhibitory ability against Arabidopsis thaliana AHAS. With molecular docking, compound 3b insert into Arabidopsis thaliana AHAS stably by an H-bond with Arg377 and cation- $\pi$ interactions with Arg377, Trp574, Tyr579. This study suggests that compound $3 \mathrm{~b}$ may serve as a potential AHAS inhibitor which can be used as a novel herbicides and provides valuable clues for the further design and optimization of AHAS inhibitors.

Key words pyrazole sulfonamide; acetohydroxy acid synthase; herbicidal activity; molecule docking

The branched-chain amino acids (BCAAs) biosynthetic pathway exists only in plants and micro-organisms, not in the bodies of mammals, and acetohydroxy acid synthase (AHAS; EC 2.2.1.6, also referred to as acetolactate synthase, ALS) plays an important role in the BCAAs biosynthetic pathway. ${ }^{1,2}$ For those reasons, made AHAS an ideal targets for the design of "green herbicides" since the mid-1980s. ${ }^{3-5)}$ There are four main categories of listed AHAS inhibitors, including: sulfonylureas, imidazolinones, pyrimidinylthio (or oxo) benzoates and triazolopyrimidine sulfonanilides. However, the frequent use of these herbicides has caused mutations in weed AHAS. ${ }^{6-9)}$ Therefore, the discovery of new drug groups with AHAS inhibitory activity is particularly urgent.

For this reason, we chose AHAS as target, and introduced pyrazole ring instead of the triazole ring in the flucarbazone molecule (Fig. 1). The attractiveness of pyrazole and its derivatives is their versatility that allows for synthesis of a series of analogues with different moieties in them, thus affecting the electronics and by extension the properties of the resultant compounds. $^{10,11)}$ It is widely used as a large number of compounds for various applications, such agrochemicals ${ }^{12-14)}$ and medicine, ${ }^{15-18)}$ due to their broad range of biological activities. Therefore, it is expected that the introduction of the pyrazole ring can yield compounds with excellent herbicidal activity.

Herein, we describe the design and synthesis of a series of pyrazole sulfonamide derivatives by introducing a pyrazole ring into the flucarbazone molecule. The in vivo herbicidal ac-

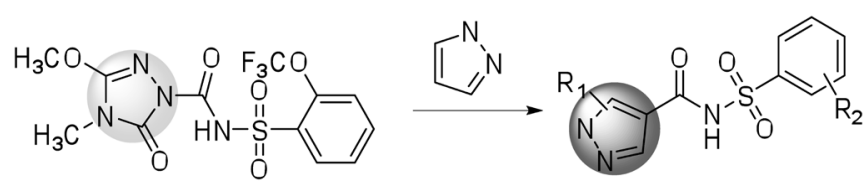

Fig. 1. Design of Target Compounds tivities of these compounds were evaluated, and subsequently molecular docking was performed for exploring the binding mode between small molecules and the Arabidopsis thaliana AHAS (AtAHAS).

\section{Results and Discussion}

Chemistry A series of novel pyrazole sulfonamide derivatives was synthesised and the general pathway is outlined in Chart 1. After cyclization of substituted phenylhydrazine<smiles>[R]c1ccc(NN)cc1</smiles><smiles>[R20]c1ccc(-n2nc(C)c(C(=O)O)c2Cl)cc1</smiles><smiles>[R2]c1ccc(-n2nc(C)c(C(=O)NS(=O)(=O)c3ccc([R])cc3)c2Cl)cc1</smiles>

Reagents and conditions: (a) $\mathrm{H}_{2} \mathrm{O}$, ethanol, $60^{\circ} \mathrm{C}$; (b) DMF, $\mathrm{POCl}_{3}, 90^{\circ} \mathrm{C}, 5 \mathrm{~h}$; (c) $\mathrm{KMnO}_{4}, 70-80^{\circ} \mathrm{C}$; (d) EDCI, HOBt, DMF, r.t.

Chart 1. General Synthesis of Compounds $\mathbf{4 a - 4 t}$ and $\mathbf{5 a}-\mathbf{5 p}$ 
Table 1. In Vivo Herbicidal Activity of the Compounds $\mathbf{3 a}-\mathbf{3 t}$

\begin{tabular}{|c|c|c|c|c|}
\hline \multirow[t]{2}{*}{ Compound } & \multirow[t]{2}{*}{$\mathrm{R}_{1}$} & \multirow[t]{2}{*}{$\mathrm{R}_{2}$} & $\begin{array}{l}\text { Rape root length } \\
\text { inhibition }(\%)\end{array}$ & \multirow{2}{*}{$\begin{array}{l}\text { CDOCKER_- } \\
\text { Interaction energy } \\
\left(\mathrm{kcal} \mathrm{mol}^{-1}\right)^{a)}\end{array}$} \\
\hline & & & $100 \mathrm{mg} / \mathrm{L}$ & \\
\hline $3 \mathbf{a}$ & $\mathrm{H}$ & $\mathrm{H}$ & 0 & -23.2624 \\
\hline $\mathbf{3 b}$ & $\mathrm{H}$ & $\mathrm{Cl}$ & 81 & -31.9225 \\
\hline $3 \mathrm{c}$ & $\mathrm{H}$ & $\mathrm{CH}_{3}$ & 12 & -24.5746 \\
\hline 3d & $\mathrm{H}$ & $\mathrm{OCH}_{3}$ & 43 & -26.8199 \\
\hline $3 e$ & $\mathrm{H}$ & $\mathrm{OCF}_{3}$ & 68 & -27.5274 \\
\hline $3 f$ & $\mathrm{~F}$ & $\mathrm{H}$ & 0 & -24.3675 \\
\hline $3 g$ & $\mathrm{~F}$ & $\mathrm{Cl}$ & 13 & -26.0059 \\
\hline $3 h$ & $\mathrm{~F}$ & $\mathrm{CH}_{3}$ & 25 & -26.6266 \\
\hline $3 \mathbf{i}$ & $\mathrm{F}$ & $\mathrm{OCH}_{3}$ & 36 & -24.7947 \\
\hline $\mathbf{3 j}$ & $\mathrm{F}$ & $\mathrm{OCF}_{3}$ & 33 & -26.9420 \\
\hline $3 \mathbf{k}$ & $\mathrm{Cl}$ & $\mathrm{H}$ & 26 & -24.0369 \\
\hline 31 & $\mathrm{Cl}$ & $\mathrm{Cl}$ & 21 & -24.6165 \\
\hline $3 m$ & $\mathrm{Cl}$ & $\mathrm{CH}_{3}$ & 16 & -26.7611 \\
\hline $3 n$ & $\mathrm{Cl}$ & $\mathrm{OCH}_{3}$ & 72 & -31.0646 \\
\hline 30 & $\mathrm{Cl}$ & $\mathrm{OCF}_{3}$ & 54 & -28.9238 \\
\hline $3 p$ & $\mathrm{CH}_{3}$ & $\mathrm{H}$ & 36 & -24.8901 \\
\hline $3 q$ & $\mathrm{CH}_{3}$ & $\mathrm{Cl}$ & 10 & -24.5399 \\
\hline $3 \mathbf{r}$ & $\mathrm{CH}_{3}$ & $\mathrm{CH}_{3}$ & 0 & -23.5403 \\
\hline $3 s$ & $\mathrm{CH}_{3}$ & $\mathrm{OCH}_{3}$ & 19 & -23.4165 \\
\hline $3 \mathbf{t}$ & $\mathrm{CH}_{3}$ & $\mathrm{OCF}_{3}$ & 46 & -24.2396 \\
\hline Monosulfuron & & & 87 & \\
\hline
\end{tabular}

a) Internal ligand strain energy and receptor-ligand interaction energy obtained from the docking study of all synthesized compounds by the CDOCKER protocol (Discovery Studio 3.1, Accelrys, Inc., San Diego, CA, U.S.A.).

and ethylacetoacetate in ethanol, 1a-1d was obtained with Vilsmeier-Haack reagent ( $N, N$-dimethylformamide (DMF)$\mathrm{POCl}_{3}$ ). Then $\mathbf{1 a}-\mathbf{1 d}$ were oxidized by $\mathrm{KMnO}_{4}$ solution to obtain $\mathbf{2 a - 2 d}$. The synthesis of the target compounds was carried out through our previously reported method. ${ }^{19)}$ All the synthetic compounds were analysed by element and spectroscopic methods, which showed that all compounds are in full accordance with the structures depicted in Table 1.

Biological Activity The in vivo herbicidal activity of the compounds $\mathbf{3 a}-\mathbf{3 t}$ was measured by using the rape root inhibition method and the measurement results are summarized in Table 1. The sulfonylurea herbicide monosulfuron was used as the control.

These results show that most of the compounds exhibited different levels of in vivo herbicidal activities at a concentration of $100 \mathrm{mg} \mathrm{L}^{-1}$. The most potent of the compounds $\mathbf{3 b}, \mathbf{3 e}$ and $3 n$ showed 81,68 and $72 \%$ inhibitory activities respectively, slightly lower than $87 \%$ inhibitory activity of monosulfuron. Furthermore, compounds 3d, 30 and 3t (43, 54, 46\%) showed about $50 \%$ inhibition activities, and the remaining compounds have $0-40 \%$ inhibitory activities. However, it is regrettable that some of the compounds showed no inhibitory activity slightest, such as compounds $\mathbf{3 a}, \mathbf{3} \mathbf{f}$ and $\mathbf{3 r}$.

According to the in vivo herbicidal activity as Table 1, three compounds tested against AtAHAS activity in vitro which in vivo herbicidal activity values of $>60 \%$ and the measurement results are summarized in Table 2. All of the compounds showed excellent inhibitory activity against AtAHAS as Table 2. Compound $\mathbf{3 e}$ showed the best inhibitory ability against AtAHAS with values of $81 \%$ at $100 \mathrm{mg} / \mathrm{L}$, compounds $\mathbf{3 b}, \mathbf{3 n}$ show greater than $60 \%$ inhibitory abilities against AtAHAS.
Table 2. In Vitro AtAHAS Inhibition of the Compounds

\begin{tabular}{cc}
\hline \hline & AtAHAS inhibition $(\%)$ \\
\cline { 2 - 2 } Compound & $100 \mathrm{mg} / \mathrm{L}$ \\
\hline 3b & 65 \\
3e & 81 \\
3n & 73 \\
Monosulfuron & 93 \\
\hline
\end{tabular}

However, the inhibitory activity of these compounds is lower than that of monosulfuron.

Molecular Docking In order to verify this assumption, molecular docking was performed by target compounds into the binding site of AtAHAS (PDB entry: $5 \mathrm{k} 6 \mathrm{t}$ ). ${ }^{20)}$ To our delight, it was clearly seen that compounds $\mathbf{3 b}$ and $\mathbf{3 n}$ showed obviously lower interaction energy than positive drugs flucarbazone, demonstrating that these compounds are likely to exhibit more potent inhibitory activity against AtAHAS (Table 1). These preliminary Computer Aided Drug Design (CADD) results suggested these design pyrazole derivatives containing sulfonamide moiety possibly served as potent herbicides by inhibiting AHAS.

We explored their binding model generated by molecular docking based on the AtAHAS (PDB entry: 5k6t) and preprocessed by the DS 3.1 (Discovery Studio 3.1, Accelrys, Inc.). As showed in Fig. 2(A), the compound $\mathbf{3 b}$ skeleton was deeply embedded into the binding pocket, suggesting the pose of $\mathbf{3 b}$ into the AtAHAS-binding site which revealed that it has suitable shape complementarity with the binding pocket, which means that the reasonableness of our design molecules. As showed in Fig. 2(B), cationic sidechain Arg377 makes cation $-\pi$ interaction with $\mathbf{3 b}$. The results of docking simulation suggested there were the same binding mode that the presence of flucarbazone molecular in a $5 \mathrm{k} 6 \mathrm{t}$ crystal protein, with electron-withdrawing group ester and sulfonyl groups on its benzene ring, still produces a cation $-\pi$ interaction with Arg377 (Figure S uploaded as an attachment). ${ }^{20)}$ In addition, Trp574 and Tyr579 are combined with electron-rich $\pi$ system of benzene and pyrazole ring in compound $\mathbf{3 b}$, another stable $\mathrm{H}$-bond was also detected in the binding model between $\mathrm{O}$ in the sulfonamide and $\operatorname{Arg} 377$ (angle $=116.9^{\circ}$, distance $=1.8 \AA$ ), which provides valuable information for the further design of AHAS inhibitors.

\section{Conclusion}

A series of novel pyrazole sulfonamide derivatives were synthesised and evaluated for their in vivo herbicidal activities and in vitro AtAHAS inhibition activities. Compound $\mathbf{3 b}$ showed the most potent in vivo herbicidal activity with values of $81 \%$ at $100 \mathrm{mg} / \mathrm{L}$, and exhibited the best inhibitory ability against AtAHAS. Docking simulation showed compound $\mathbf{3 b}$ skeleton was deeply embedded into the binding pocket. Above all, the results obtained from this study suggest that compound 3b may serve as a potential AHAS inhibitors which can be used as a novel herbicides and provide valuable information for the design AHAS inhibitors.

\section{Experimental}

Materials and Measurements All chemicals (reagent grade) used were purchased from Sigma-Aldrich (St. Louis, 


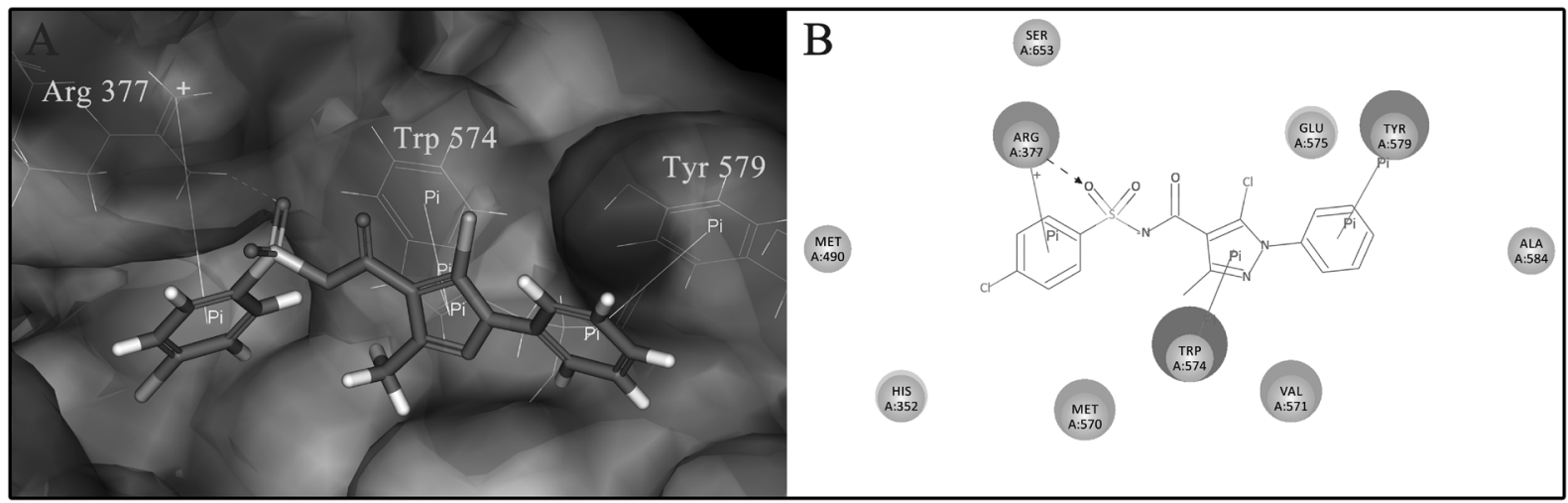

Fig. 2. Binding Model of $\mathbf{3 b}$ in the Active Site of AtAHAS (PDB Entry: 5k6t)

The $\mathrm{H}$-bond is displayed as dashed line and $\pi$ interaction is displayed as solid line.

MO, U.S.A.) and Sinopharm Chemical Reagent Co., Ltd. (Beijing, China). All the ${ }^{1} \mathrm{H}-\mathrm{NMR}$ spectra were measured on an Agilent DD2 $600 \mathrm{~Hz}$ spectrometer (Agilent Technologies Corporation, Santa Clara, CA, U.S.A.). Chemical shifts were reported in ppm $(\delta)$. Electrospray ionization (ESI)-MS spectra were recorded on a Mariner System 5304 mass spectrometer (Applied Biosystems, Foster City, CA, U.S.A.). Elemental analyses were performed on a CHN-O-Rapid instrument (Leco, Tres Cantos, Madrid, Spain) and were within $0.4 \%$ of the theoretical values. Melting points were measured without correction.

General Procedure for Synthesis of 5-Chloro-1-aryl3-methyl-1H-pyrazole-4-carboxylic Acids 2a-2d ${ }^{21)}$ paraSubstituted phenyl hydrazine $(25 \mathrm{mmol})$ was dissolved in anhydrous ethanol, ethyl acetoacetate $(25 \mathrm{mmol})$ was slowly added and stirred at $70-80^{\circ} \mathrm{C}$ for $5 \mathrm{~h}$, then the anhydrous ethanol was removed under reduced pressure to form a solid, which was dissolved in DMF $(20 \mathrm{~mL})$ and phosphorus oxychloride $(16 \mathrm{~mL})$ of cold mixed solution and stirred at $90^{\circ} \mathrm{C}$ for $1 \mathrm{~h}$. The resulting mixture was poured into ice-cold water, the resulting solid was separated by filtration to give the light yellow solid. Then above product was oxidized by $5.0 \mathrm{~mol} / \mathrm{L}$ $\mathrm{KMnO}_{4}$ solution, stirred at $70-80^{\circ} \mathrm{C}$. After cooling to room temperature the $\mathrm{pH}$ of the reaction mixture was adjusted to $\mathrm{pH} 7-8$ by the dropwise addition of $3.0 \mathrm{~mol} / \mathrm{L} \mathrm{KOH}$ solution, and the solution was filtered, $6.0 \mathrm{~mol} / \mathrm{L} \mathrm{HCl}$ solution was added to the solution and solid $\mathbf{2 a - 2 d}$ eventually separated out. The crude product obtained was recrystallized from anhydrous ethanol to afford the pure product.

General Procedure for Synthesis of 5-Chloro-3-methyl1-phenyl- $N$-(phenylsulfonyl)- $1 H$-pyrazole-4-carboxamide 3a-3t To a stirred solution of the intermediates compound 2a-2d (1 mmol) with triethylamine $(2 \mathrm{mmol})$ into DMF $(12 \mathrm{~mL})$, then a mixture of 1-ethyl-(3-(3-dimethylamino)propyl)-carbodiimide hydrochloride (EDCI) (1 mmol) and $N$-hydroxybenzotriazole (HOBt) $(1 \mathrm{mmol})$ was placed in the reaction system, stirred at room temperature for $30 \mathrm{~min}$, the mixture of substituted benzene sulfonamide $(1 \mathrm{mmol})$ and DMF $(5 \mathrm{~mL})$ was added in the reaction system, the reaction mixture was monitored by TLC. After completion of the reaction, the product was extracted from chloroform with water, $0.2 \mathrm{~mol} / \mathrm{L}$ hydrochloric acid, water, $2.0 \mathrm{~mol} / \mathrm{L}$ sodium hydroxide, saturated sodium chloride successively, and then dried, concentrated, and purified by preparative thin layer chroma- tography followed by recrystallization from ethanol.

5-Chloro-3-methyl-1-phenyl- $N$-(phenylsulfonyl)- $1 H$ pyrazole-4-carboxamide (3a)

Light yellow solid, yield $74 \%$; mp $156-158^{\circ} \mathrm{C}$; ${ }^{1} \mathrm{H}-\mathrm{NMR}$ $\left(600 \mathrm{MHz}, \mathrm{CDCl}_{3}\right) \delta: 8.65(\mathrm{~s}, 1 \mathrm{H}), 8.17(\mathrm{~d}, J=8.0 \mathrm{~Hz}, 2 \mathrm{H})$, 7.67 (t, $J=7.4 \mathrm{~Hz}, 1 \mathrm{H}), 7.58(\mathrm{t}, J=7.7 \mathrm{~Hz}, 2 \mathrm{H}), 7.44$ (dd, $J=8.6$, $4.7 \mathrm{~Hz}, 3 \mathrm{H}), 7.21(\mathrm{t}, J=8.3 \mathrm{~Hz}, 2 \mathrm{H}), 2.47$ (s, 3H). ${ }^{13} \mathrm{C}-\mathrm{NMR}$ $(151 \mathrm{MHz}$, dimethyl sulfoxide (DMSO)) $\delta: 163.17,150.21$, $140.81,138.04,133.63,131.93,130.07,129.02,128.43,128.31$, 127.34, 116.83, 13.72. MS (ESI): $376.8 \quad\left(\mathrm{C}_{17} \mathrm{H}_{14} \mathrm{ClN}_{3} \mathrm{O}_{3} \mathrm{~S}\right.$, $\left.[\mathrm{M}+\mathrm{H}]^{+}\right)$. Anal. Calcd for $\mathrm{C}_{17} \mathrm{H}_{14} \mathrm{ClN}_{3} \mathrm{O}_{3} \mathrm{~S}: \mathrm{C}, 54.33 ; \mathrm{H}, 3.75$; N, 11.18. Found: C, 54.36; H, 3.79; N, 11.25.

5-Chloro- $N$-((4-chlorophenyl) sulfonyl)-3-methyl-1phenyl-1H-pyrazole-4-carboxamide (3b)

Light yellow solid, yield $73 \%$; mp $152-154^{\circ} \mathrm{C}$; ${ }^{1} \mathrm{H}-\mathrm{NMR}$ $\left(600 \mathrm{MHz}, \mathrm{CDCl}_{3}\right) \delta: 8.65(\mathrm{~s}, 1 \mathrm{H}), 8.10(\mathrm{~d}, J=8.6 \mathrm{~Hz}, 2 \mathrm{H})$, $7.55(\mathrm{~d}, J=8.6 \mathrm{~Hz}, 2 \mathrm{H}), 7.44(\mathrm{dd}, J=8.9,4.7 \mathrm{~Hz}, 3 \mathrm{H}), 7.21(\mathrm{t}$, $J=8.4 \mathrm{~Hz}, 2 \mathrm{H}), 2.48(\mathrm{~s}, 3 \mathrm{H}) .{ }^{13} \mathrm{C}-\mathrm{NMR}(151 \mathrm{MHz}, \mathrm{DMSO})$ $\delta: 163.20,150.20,139.08,138.82,138.02,133.63,130.04$, 129.79, 129.57, 128.40, 128.34, 116.80, 13.73. MS (ESI): 411.2 $\left(\mathrm{C}_{17} \mathrm{H}_{13} \mathrm{Cl}_{2} \mathrm{~N}_{3} \mathrm{O}_{3} \mathrm{~S}\right.$, $\left.[\mathrm{M}+\mathrm{H}]^{+}\right)$. Anal. Calcd for $\mathrm{C}_{17} \mathrm{H}_{13} \mathrm{Cl}_{2} \mathrm{~N}_{3} \mathrm{O}_{3} \mathrm{~S}$ : C, 49.77; H, 3.19; N, 10.24. Found: C, 49.79; H, 3.24; N, 10.31.

5-Chloro-3-methyl-1-phenyl- $N$-tosyl-1 $H$-pyrazole-4carboxamide (3c)

Light yellow solid, yield $71 \%$; mp $131-134^{\circ} \mathrm{C}$; ${ }^{1} \mathrm{H}-\mathrm{NMR}$ $\left(600 \mathrm{MHz}, \mathrm{CDCl}_{3}\right) \delta: 8.67(\mathrm{~s}, 1 \mathrm{H}), 8.03(\mathrm{~d}, J=8.3 \mathrm{~Hz}, 2 \mathrm{H})$, 7.44 (dd, $J=8.9,4.7 \mathrm{~Hz}, 3 \mathrm{H}), 7.37$ (d, $J=8.1 \mathrm{~Hz}, 2 \mathrm{H}), 7.20$ (t, $J=8.5 \mathrm{~Hz}, 2 \mathrm{H}), 2.47(\mathrm{~s}, 3 \mathrm{H}), 2.45(\mathrm{~s}, 3 \mathrm{H}) .{ }^{13} \mathrm{C}-\mathrm{NMR}(151 \mathrm{MHz}$, DMSO) $\delta: 163.17,150.04,137.99,137.81,137.64,133.65$, 130.02, 129.33, 128.37, 128.36, 128.11, 116.80, 21.56, 13.70 . MS (ESI): $390.8\left(\mathrm{C}_{18} \mathrm{H}_{16} \mathrm{ClN}_{3} \mathrm{O}_{3} \mathrm{~S},[\mathrm{M}+\mathrm{H}]^{+}\right)$. Anal. Calcd for $\mathrm{C}_{18} \mathrm{H}_{16} \mathrm{ClN}_{3} \mathrm{O}_{3} \mathrm{~S}$ : C, 55.46; H, 4.14; N, 10.78. Found: C, 55.50; $\mathrm{H}, 4.19 ; \mathrm{N}, 10.72$.

5-Chloro- $N$-((4-methoxyphenyl)sulfonyl)-3-methyl-1phenyl- $1 H$-pyrazole-4-carboxamide (3d)

Light yellow solid, yield $84 \%$; mp $113-114^{\circ} \mathrm{C}$; ${ }^{1} \mathrm{H}-\mathrm{NMR}$ $\left(600 \mathrm{MHz}, \mathrm{CDCl}_{3}\right) \delta: 8.71(\mathrm{~s}, 1 \mathrm{H}), 8.23(\mathrm{~d}, J=8.9 \mathrm{~Hz}, 2 \mathrm{H})$, $7.57-7.47(\mathrm{~m}, 3 \mathrm{H}), 7.45(\mathrm{~d}, J=6.8 \mathrm{~Hz}, 2 \mathrm{H}), 7.39$ (d, $J=8.7 \mathrm{~Hz}$, 2H), 2.49 (s, 3H). ${ }^{13} \mathrm{C}-\mathrm{NMR}(151 \mathrm{MHz}, \mathrm{DMSO}) \delta$ : 163.84, $163.17,150.07,133.21,129.55,129.38,128.39,126.13,116.87$, 116.72, 114.62, 56.81, 13.70. MS (ESI): $406.8\left(\mathrm{C}_{18} \mathrm{H}_{16} \mathrm{ClN}_{3} \mathrm{O}_{4} \mathrm{~S}\right.$, $\left.[\mathrm{M}+\mathrm{H}]^{+}\right)$. Anal. Calcd for $\mathrm{C}_{18} \mathrm{H}_{16} \mathrm{ClN}_{3} \mathrm{O}_{4} \mathrm{~S}: \mathrm{C}, 53.27 ; \mathrm{H}, 3.97$; N, 10.35. Found: C, 53.31; H, 4.02; N, 10.28. 
5-Chloro-3-methyl-1-phenyl- $N$-((4-(trifluoromethoxy)phenyl)sulfonyl)- $1 H$-pyrazole-4-carboxamide (3e)

Light yellow solid, yield $79 \%$; mp $117-119^{\circ} \mathrm{C}$; ${ }^{1} \mathrm{H}-\mathrm{NMR}$ $\left(600 \mathrm{MHz}, \mathrm{CDCl}_{3}\right) \delta: 8.71(\mathrm{~s}, 1 \mathrm{H}), 8.23(\mathrm{~d}, J=8.6 \mathrm{~Hz}, 2 \mathrm{H})$, $7.54-7.48(\mathrm{~m}, 3 \mathrm{H}), 7.45(\mathrm{~d}, J=7.3 \mathrm{~Hz}, 2 \mathrm{H}), 7.39$ (d, $J=8.4 \mathrm{~Hz}$, 2H), 2.49 (s, 3H). ${ }^{13} \mathrm{C}-\mathrm{NMR}(151 \mathrm{MHz}, \mathrm{DMSO}) \delta: 163.17$, $153.83, \quad 150.04, \quad 133.13,129.76, \quad 129.55,128.39,129.38$, 126.17, 116.87, 116.72, 114.65, 13.71. MS (ESI): 460.8 $\left(\mathrm{C}_{18} \mathrm{H}_{13} \mathrm{ClF}_{3} \mathrm{~N}_{3} \mathrm{O}_{4} \mathrm{~S},[\mathrm{M}+\mathrm{H}]^{+}\right)$. Anal. Calcd for $\mathrm{C}_{18} \mathrm{H}_{13} \mathrm{ClF}_{3} \mathrm{~N}_{3} \mathrm{O}_{4} \mathrm{~S}$ : C, 47.02; H, 2.85; N, 9.14. Found: C, 47.11; H, 2.88; N, 9.21.

5-Chloro-1-(4-fluorophenyl)-3-methyl- $\mathrm{N}$-(phenylsulfonyl)- $1 \mathrm{H}$ pyrazole-4-carboxamide (3f)

Light yellow solid, yield $80 \%$; mp $158-160^{\circ} \mathrm{C}$; ${ }^{1} \mathrm{H}-\mathrm{NMR}$ $\left(600 \mathrm{MHz}, \mathrm{CDCl}_{3}\right) \delta: 8.65(\mathrm{~s}, 1 \mathrm{H}), 8.16(\mathrm{~d}, J=8.6 \mathrm{~Hz}, 2 \mathrm{H})$, 7.67 (t, $J=7.5 \mathrm{~Hz}, 1 \mathrm{H}), 7.58$ (t, $J=7.8 \mathrm{~Hz}, 2 \mathrm{H}), 7.44$ (dd, $J=8.9$, $4.7 \mathrm{~Hz}, 2 \mathrm{H}), 7.21(\mathrm{t}, J=8.5 \mathrm{~Hz}, 2 \mathrm{H}), 2.47$ (s, 3H). ${ }^{13} \mathrm{C}-\mathrm{NMR}$ (151 MHz, DMSO) $\delta: 163.17,160.47,150.22,139.08,138.82$, 133.63, 132.81, 129.79, 129.57, 116.84, 116.12, 115.63, 13.70. MS (ESI): $394.8\left(\mathrm{C}_{17} \mathrm{H}_{13} \mathrm{ClFN}_{3} \mathrm{O}_{3} \mathrm{~S},[\mathrm{M}+\mathrm{H}]^{+}\right)$. Anal. Calcd for $\mathrm{C}_{17} \mathrm{H}_{13} \mathrm{ClFN}_{3} \mathrm{O}_{3} \mathrm{~S}$ : C, 51.85; H, 3.33; N, 10.67. Found: C, 51.90; $\mathrm{H}, 3.38 ; \mathrm{N}, 10.72$.

5-Chloro- $N$-((4-chlorophenyl)sulfonyl)-1-(4-fluorophenyl)-3methyl-1H-pyrazole-4-carboxamide $(\mathbf{3 g})$

Light yellow solid, yield $81 \%$; mp $157-159^{\circ} \mathrm{C}$; ${ }^{1} \mathrm{H}-\mathrm{NMR}$ $\left(600 \mathrm{MHz}, \mathrm{CDCl}_{3}\right) \delta: 8.67 \quad(\mathrm{~s}, 1 \mathrm{H}), 8.10 \quad(\mathrm{~d}, J=8.6 \mathrm{~Hz}$, $2 \mathrm{H}), 7.55$ (d, $J=8.6 \mathrm{~Hz}, 2 \mathrm{H}), 7.44$ (dd, $J=8.9,4.7 \mathrm{~Hz}, 2 \mathrm{H})$, $7.21(\mathrm{t}, J=8.4 \mathrm{~Hz}, 2 \mathrm{H}), 2.47(\mathrm{~s}, 3 \mathrm{H}) .{ }^{13} \mathrm{C}-\mathrm{NMR}(151 \mathrm{MHz}$, DMSO) $\delta: 163.17,160.45,150.21,138.94,137.52,133.63$, $132.81,129.17,128.75,116.80,116.18,115.67,13.71 . \mathrm{MS}$ (ESI): $429.2\left(\mathrm{C}_{17} \mathrm{H}_{12} \mathrm{Cl}_{2} \mathrm{FN}_{3} \mathrm{O}_{3} \mathrm{~S},[\mathrm{M}+\mathrm{H}]^{+}\right)$. Anal. Calcd for $\mathrm{C}_{17} \mathrm{H}_{12} \mathrm{Cl}_{2} \mathrm{FN}_{3} \mathrm{O}_{3} \mathrm{~S}$ : C, 47.68; H, 2.82; N, 9.81. Found: C, 47.73; $\mathrm{H}, 2.89 ; \mathrm{N}, 9.76$.

5-Chloro-1-(4-fluorophenyl)-3-methyl- $N$-tosyl-1 $H$-pyrazole-4carboxamide (3h)

Light yellow solid, yield $69 \%$; mp $160-162^{\circ} \mathrm{C}$; ${ }^{1} \mathrm{H}-\mathrm{NMR}$ $\left(600 \mathrm{MHz}, \mathrm{CDCl}_{3}\right) \delta: 8.62(\mathrm{~s}, 1 \mathrm{H}), 8.04(\mathrm{~d}, J=8.3 \mathrm{~Hz}, 2 \mathrm{H})$, $7.48-7.41(\mathrm{~m}, 2 \mathrm{H}), 7.37(\mathrm{~d}, J=8.1 \mathrm{~Hz}, 2 \mathrm{H}), 7.20$ (t, $J=8.5 \mathrm{~Hz}$, 2H), 2.47 (s, 3H), 2.45 (s, 3H). ${ }^{13} \mathrm{C}-\mathrm{NMR}$ (151 MHz, DMSO) $\delta$ : 163.16, 160.55, 150.24, 137.81, 137.63, 133.63, 132.81, 129.34, 128.37, 116.81, 116.13, 115.61, 13.72. MS (ESI): 408.8 $\left(\mathrm{C}_{18} \mathrm{H}_{15} \mathrm{ClFN}_{3} \mathrm{O}_{3} \mathrm{~S},[\mathrm{M}+\mathrm{H}]^{+}\right)$. Anal. Calcd for $\mathrm{C}_{18} \mathrm{H}_{15} \mathrm{ClFN}_{3} \mathrm{O}_{3} \mathrm{~S}$ : C, 53.01; H, 3.71; N, 10.30. Found: C, 53.09; H, 3.75; N, 10.26.

5-Chloro-1-(4-fluorophenyl)- $N$-((4-methoxy phenyl)sulfonyl)-3-methyl-1 $H$-pyrazole-4-carboxamide (3i)

Light yellow solid, yield $66 \%$; mp $114-116^{\circ} \mathrm{C}$; ${ }^{1} \mathrm{H}-\mathrm{NMR}$ $\left(600 \mathrm{MHz}, \mathrm{CDCl}_{3}\right) \delta: 8.62(\mathrm{~s}, 1 \mathrm{H}), 8.09(\mathrm{~d}, J=8.9 \mathrm{~Hz}, 2 \mathrm{H})$, $7.47-7.40(\mathrm{~m}, 2 \mathrm{H}), 7.20(\mathrm{t}, J=8.4 \mathrm{~Hz}, 2 \mathrm{H}), 7.02(\mathrm{~d}, J=8.9 \mathrm{~Hz}$, 2H), 3.89 (s, 3H), 2.48 (s, 3H). ${ }^{13} \mathrm{C}-\mathrm{NMR}$ (151 MHz, DMSO) $\delta 163.81,163.21,160.41,150.23,133.63,133.12,132.81,126.12$, $116.84,116.18,115.63,114.63,55.83,13.72$. MS (ESI): 424.8 $\left(\mathrm{C}_{18} \mathrm{H}_{15} \mathrm{ClFN}_{3} \mathrm{O}_{4} \mathrm{~S},[\mathrm{M}+\mathrm{H}]^{+}\right)$. Anal. Calcd for $\mathrm{C}_{18} \mathrm{H}_{15} \mathrm{ClFN}_{3} \mathrm{O}_{4} \mathrm{~S}$ : C, 51.01; H, 3.57; N, 9.91. Found: C, 51.08; H, 3.62; N, 9.97.

5-Chloro-1-(4-fluorophenyl)-3-methyl- $N$-((4-(trifluoromethoxy)phenyl)sulfonyl)-1H-pyrazole-4-carboxamide (3j)

Light yellow solid, yield $63 \%$; mp $133-135^{\circ} \mathrm{C}$; ${ }^{1} \mathrm{H}-\mathrm{NMR}$ $\left(600 \mathrm{MHz}, \mathrm{CDCl}_{3}\right) \delta: 8.70(\mathrm{~s}, 1 \mathrm{H}), 8.23(\mathrm{~d}, J=8.8 \mathrm{~Hz}$, $2 \mathrm{H}), 7.48-7.42(\mathrm{~m}, 2 \mathrm{H}), 7.39(\mathrm{~d}, J=8.4 \mathrm{~Hz}, 2 \mathrm{H}), 7.21$ (t, $J=8.4 \mathrm{~Hz}, 2 \mathrm{H}), 2.48(\mathrm{~s}, 3 \mathrm{H}),{ }^{13} \mathrm{C}-\mathrm{NMR}(151 \mathrm{MHz}, \mathrm{DMSO})$ $\delta: 163.20,160.41,153.84,150.20,133.63,133.14,132.81$, $129.74,126.15,116.85,116.18,115.67,114.67,13.73 . \mathrm{MS}$ (ESI): $478.8\left(\mathrm{C}_{18} \mathrm{H}_{12} \mathrm{ClF}_{4} \mathrm{~N}_{3} \mathrm{O}_{4} \mathrm{~S},[\mathrm{M}+\mathrm{H}]^{+}\right)$. Anal. Calcd for
$\mathrm{C}_{18} \mathrm{H}_{12} \mathrm{ClF}_{4} \mathrm{~N}_{3} \mathrm{O}_{4} \mathrm{~S}: \mathrm{C}, 45.25 ; \mathrm{H}, 2.53 ; \mathrm{N}, 8.79$. Found: C, 45.33; $\mathrm{H}, 2.55$; N, 8.74.

5-Chloro-1-(4-chlorophenyl)-3-methyl-N-(phenylsulfonyl)-1 $H$-pyrazole-4-carboxamide (3k)

Light yellow solid, yield $75 \%$; mp $168-170^{\circ} \mathrm{C}$; ${ }^{1} \mathrm{H}-\mathrm{NMR}$ $\left(600 \mathrm{MHz}, \mathrm{CDCl}_{3}\right) \delta: 8.66(\mathrm{~s}, 1 \mathrm{H}), 8.16(\mathrm{~d}, J=7.5 \mathrm{~Hz}, 2 \mathrm{H}), 7.67$ $(\mathrm{t}, J=7.5 \mathrm{~Hz}, 1 \mathrm{H}), 7.58(\mathrm{t}, J=7.8 \mathrm{~Hz}, 2 \mathrm{H}), 7.49(\mathrm{~d}, J=8.7 \mathrm{~Hz}$, $2 \mathrm{H}), 7.41(\mathrm{~d}, J=8.7 \mathrm{~Hz}, 2 \mathrm{H}), 2.47(\mathrm{~s}, 3 \mathrm{H}) .{ }^{13} \mathrm{C}-\mathrm{NMR}(151 \mathrm{MHz}$, DMSO) $\delta$ : $163.17,150.21,139.08,138.82,135.32,133.63$, $131.51,129.79,129.57,129.45,119.87,116.84,13.70 . \mathrm{MS}$ (ESI): $411.2\left(\mathrm{C}_{18} \mathrm{H}_{12} \mathrm{ClF}_{4} \mathrm{~N}_{3} \mathrm{O}_{4} \mathrm{~S},[\mathrm{M}+\mathrm{H}]^{+}\right)$. Anal. Calcd for $\mathrm{C}_{18} \mathrm{H}_{12} \mathrm{ClF}_{4} \mathrm{~N}_{3} \mathrm{O}_{4} \mathrm{~S}: \mathrm{C}, 49.77 ; \mathrm{H}, 3.19 ; \mathrm{N}, 10.24$. Found: C, 49.80; $\mathrm{H}, 3.14 ; \mathrm{N}, 10.30$.

5-Chloro-1-(4-chlorophenyl)- $N$-((4-chlorophenyl)sulfonyl)-3methyl-1H-pyrazole-4-carboxamide (3l)

Light yellow solid, yield $76 \%$; mp $174-176^{\circ} \mathrm{C}$; ${ }^{1} \mathrm{H}-\mathrm{NMR}$ $\left(600 \mathrm{MHz} \mathrm{CDCl}_{3}\right) \delta: 8.67(\mathrm{~s}, 1 \mathrm{H}), 8.10(\mathrm{~d}, J=8.6 \mathrm{~Hz}, 2 \mathrm{H}), 7.55$ (d, $J=8.6 \mathrm{~Hz}, 2 \mathrm{H}), 7.49$ (d, $J=8.7 \mathrm{~Hz}, 2 \mathrm{H}), 7.42$ (d, $J=8.7 \mathrm{~Hz}$, 2H), 2.47 (s, 3H). ${ }^{13} \mathrm{C}-\mathrm{NMR}(151 \mathrm{MHz}, \mathrm{DMSO}) \delta: 163.17$, $150.27,138.93,137.56,135.32,133.70,131.54,129.47,129.15$, 128.76, 119.84, 116.75, 13.70. MS (ESI): $445.7\left(\mathrm{C}_{17} \mathrm{H}_{12} \mathrm{Cl}_{3} \mathrm{~N}_{3} \mathrm{O}_{3} \mathrm{~S}\right.$, $\left.[\mathrm{M}+\mathrm{H}]^{+}\right)$. Anal. Calcd for $\mathrm{C}_{17} \mathrm{H}_{12} \mathrm{Cl}_{3} \mathrm{~N}_{3} \mathrm{O}_{3} \mathrm{~S}: \mathrm{C}, 45.91 ; \mathrm{H}, 2.72$; $\mathrm{N}, 9.45$. Found: C, 45.97; H, 2.77; N, 9.51.

5-Chloro-1-(4-chlorophenyl)-3-methyl- $N$-tosyl- $1 H$ pyrazole-4-carboxamide $(3 \mathrm{~m})$

Light yellow solid, yield $72 \%$; mp $155-157^{\circ} \mathrm{C} ;{ }^{1} \mathrm{H}-\mathrm{NMR}$ $\left(600 \mathrm{MHz}, \mathrm{CDCl}_{3}\right) \delta: 8.62(\mathrm{~s}, 1 \mathrm{H}), 8.04(\mathrm{~d}, J=8.1 \mathrm{~Hz}, 2 \mathrm{H}), 7.49$ $(\mathrm{d}, J=8.6 \mathrm{~Hz}, 2 \mathrm{H}), 7.41(\mathrm{~d}, J=8.6 \mathrm{~Hz}, 2 \mathrm{H}), 7.37(\mathrm{~d}, J=8.1 \mathrm{~Hz}$, 2H), 2.47 (s, 3H), 2.45 (s, 3H). ${ }^{13} \mathrm{C}-\mathrm{NMR}$ (151 MHz, DMSO) $\delta$ : $163.21,150.22,137.89,137.65,135.37,133.63,131.56,129.45$, 129.37, 128.36, 119.84, 116.81, 21.34, 13.73. MS (ESI): 425.3 $\left(\mathrm{C}_{18} \mathrm{H}_{15} \mathrm{Cl}_{2} \mathrm{~N}_{3} \mathrm{O}_{3} \mathrm{~S},[\mathrm{M}+\mathrm{H}]^{+}\right)$. Anal. Calcd for $\mathrm{C}_{18} \mathrm{H}_{15} \mathrm{Cl}_{2} \mathrm{~N}_{3} \mathrm{O}_{3} \mathrm{~S}$ : C, 50.95; H, 3.56; N, 9.90. Found: C, 50.92; H, 3.63; N, 9.96.

5-Chloro-1-(4-chlorophenyl)- $N$-((4-methoxy phenyl)sulfonyl)-3-methyl-1 $H$-pyrazole-4-carboxamide (3n)

Light yellow solid, yield $85 \%$; mp $167-169^{\circ} \mathrm{C}$; ${ }^{1} \mathrm{H}-\mathrm{NMR}$ $\left(600 \mathrm{MHz}, \mathrm{CDCl}_{3}\right) \delta: 8.63(\mathrm{~s}, 1 \mathrm{H}), 8.09(\mathrm{~d}, J=9.0 \mathrm{~Hz}, 2 \mathrm{H}), 7.49$ $(\mathrm{d}, J=8.8 \mathrm{~Hz}, 2 \mathrm{H}), 7.41(\mathrm{~d}, J=8.8 \mathrm{~Hz}, 2 \mathrm{H}), 7.02(\mathrm{~d}, J=9.0 \mathrm{~Hz}$, 2H), 3.89 (s, 3H), 2.47 (s, 3H). ${ }^{13} \mathrm{C}-\mathrm{NMR}$ (151 MHz, DMSO) $\delta$ : $163.84,163.24,150.27,135.32,133.66,133.12,131.45,129.45$, 126.15, 119.84, 116.14, 114.63, 55.84, 13.76. MS (ESI): 441.3 $\left(\mathrm{C}_{18} \mathrm{H}_{15} \mathrm{Cl}_{2} \mathrm{~N}_{3} \mathrm{O} \mathrm{O}_{4}\right.$, $\left.[\mathrm{M}+\mathrm{H}]^{+}\right)$. Anal. Calcd for $\mathrm{C}_{18} \mathrm{H}_{15} \mathrm{Cl}_{2} \mathrm{~N}_{3} \mathrm{O}_{4} \mathrm{~S}$ : C, 49.10; H, 3.43; N, 9.54. Found: C, 49.18; H, 3.48; N, 9.59.

5 - Chloro-1 - (4 - chlorophenyl)-3-methyl-N-((4(trifluoromethoxy)phenyl)sulfonyl)-1H-pyrazole-4carboxamide (3o)

Light yellow solid, yield $86 \%$; mp $158-160^{\circ} \mathrm{C}$; ${ }^{1} \mathrm{H}-\mathrm{NMR}$ $\left(600 \mathrm{MHz}, \mathrm{CDCl}_{3}\right) \delta: 8.69(\mathrm{~s}, 1 \mathrm{H}), 8.23(\mathrm{~d}, J=8.9 \mathrm{~Hz}, 2 \mathrm{H}), 7.49$ (d, $J=8.7 \mathrm{~Hz}, 2 \mathrm{H}), 7.40$ (dd, $J=13.8,8.7 \mathrm{~Hz}, 4 \mathrm{H}), 2.48$ (s, 3H). ${ }^{13} \mathrm{C}-\mathrm{NMR}$ (151 MHz, DMSO) $\delta: 163.26,153.83,150.27,135.34$, $133.71,133.16,131.54,129.74,129.45,126.19,119.87,116.60$, 114.67, 13.70. MS (ESI): $495.2\left(\mathrm{C}_{18} \mathrm{H}_{12} \mathrm{Cl}_{2} \mathrm{~F}_{3} \mathrm{~N}_{3} \mathrm{O}_{4} \mathrm{~S},[\mathrm{M}+\mathrm{H}]^{+}\right)$. Anal. Calcd for $\mathrm{C}_{18} \mathrm{H}_{12} \mathrm{Cl}_{2} \mathrm{~F}_{3} \mathrm{~N}_{3} \mathrm{O}_{4} \mathrm{~S}$ : C, 43.74; H, 2.45; N, 8.50. Found: C, 43.78; H, 2.47; N, 8.57.

5-Chloro-3-methyl- $N$-(phenylsulfonyl)-1-( $p$-tolyl $)-1 H$ pyrazole-4-carboxamide (3p)

Light yellow solid, yield $88 \%$; mp $184-186^{\circ} \mathrm{C}$; ${ }^{1} \mathrm{H}-\mathrm{NMR}$ $\left(600 \mathrm{MHz}, \mathrm{CDCl}_{3}\right) \delta: 8.69(\mathrm{~s}, 1 \mathrm{H}), 8.16(\mathrm{~d}, J=7.4 \mathrm{~Hz}, 2 \mathrm{H}), 7.66$ (t, $J=7.5 \mathrm{~Hz}, 1 \mathrm{H}), 7.58(\mathrm{t}, J=7.8 \mathrm{~Hz}, 2 \mathrm{H}), 7.34-7.28(\mathrm{~m}, 4 \mathrm{H})$, $2.47(\mathrm{~s}, 3 \mathrm{H}), 2.43(\mathrm{~s}, 3 \mathrm{H}) .{ }^{13} \mathrm{C}-\mathrm{NMR}(151 \mathrm{MHz}, \mathrm{DMSO}) \delta$ : $163.24,150.27,139.08,138.82,135.84,134.26,133.57,129.79$, 
129.67, 129.57, 125.45, 116.59, 21.34, 13.70. MS (ESI): 390.8 $\left(\mathrm{C}_{18} \mathrm{H}_{16} \mathrm{ClN}_{3} \mathrm{O}_{3} \mathrm{~S},[\mathrm{M}+\mathrm{H}]^{+}\right)$. Anal. Calcd for $\mathrm{C}_{18} \mathrm{H}_{16} \mathrm{ClN}_{3} \mathrm{O}_{3} \mathrm{~S}: \mathrm{C}$, 55.46; H, 4.14; N, 10.78. Found: C, 55.52; H, 4.19; N, 10.75 .

5-Chloro- $N$-((4-chlorophenyl)sulfonyl)-3-methyl-1-( $p$ tolyl)- $1 H$-pyrazole-4-carboxamide (3q)

Light yellow solid, yield $84 \%$; mp $114-116^{\circ} \mathrm{C}$; ${ }^{1} \mathrm{H}-\mathrm{NMR}$ $\left(600 \mathrm{MHz}, \mathrm{CDCl}_{3}\right) \delta: 8.70(\mathrm{~s}, 1 \mathrm{H}), 8.10(\mathrm{~d}, J=8.2 \mathrm{~Hz}, 2 \mathrm{H})$, 7.54 (d, $J=8.4 \mathrm{~Hz}, 3 \mathrm{H}), 7.31(\mathrm{~d}, J=1.9 \mathrm{~Hz}, 3 \mathrm{H}), 2.47$ (s, 3H), 2.43 (s, 3H). ${ }^{13} \mathrm{C}-\mathrm{NMR}$ (151 MHz, DMSO) $\delta$ : 163.17, 150.31, 138.96, 137.54, 135.94, 134.26, 133.63, 129.57, 129.16, 128.71, 125.13, 116.80, 21.33, 13.73. MS (ESI): $425.3\left(\mathrm{C}_{18} \mathrm{H}_{15} \mathrm{Cl}_{2} \mathrm{~N}_{3} \mathrm{O}_{3} \mathrm{~S}\right.$, $\left.[\mathrm{M}+\mathrm{H}]^{+}\right)$. Anal. Calcd for $\mathrm{C}_{18} \mathrm{H}_{15} \mathrm{Cl}_{2} \mathrm{~N}_{3} \mathrm{O}_{3} \mathrm{~S}: \mathrm{C}, 50.95 ; \mathrm{H}, 3.56$; N, 9.90. Found: C, 51.02; H, 3.53; N, 9.98 .

5-Chloro-3-methyl-1-( $p$-tolyl)- $N$-tosyl-1 $H$-pyrazole-4carboxamide (3r)

Light yellow solid, yield $76 \%$; mp $80-83^{\circ} \mathrm{C}$; ${ }^{1} \mathrm{H}-\mathrm{NMR}$ $\left(600 \mathrm{MHz}, \mathrm{CDCl}_{3}\right) \delta: 8.68(\mathrm{~s}, 1 \mathrm{H}), 8.03(\mathrm{~d}, J=8.2 \mathrm{~Hz}, 2 \mathrm{H}), 7.36$ (d, $J=8.1 \mathrm{~Hz}, 3 \mathrm{H}), 7.31(\mathrm{~d}, J=4.7 \mathrm{~Hz}, 3 \mathrm{H}), 2.47(\mathrm{~s}, 3 \mathrm{H}), 2.45$ (s, 3H), 2.43 (s, 3H). ${ }^{13} \mathrm{C}-\mathrm{NMR}(151 \mathrm{MHz}, \mathrm{DMSO}) \delta: 163.20$, $150.24,137.84,137.65,135.94,134.26,133.63,129.67,129.31$, 128.37, 125.13, 116.80, 21.37, 21.57, 13.70. MS (ESI): 404.8 $\left(\mathrm{C}_{19} \mathrm{H}_{18} \mathrm{ClN}_{3} \mathrm{O}_{3} \mathrm{~S},[\mathrm{M}+\mathrm{H}]^{+}\right)$. Anal. Calcd for $\mathrm{C}_{19} \mathrm{H}_{18} \mathrm{ClN}_{3} \mathrm{O}_{3} \mathrm{~S}: \mathrm{C}$, 56.50; H, 4.49; N, 10.40. Found: C, 56.57; H, 4.53; N, 10.35 .

5-Chloro- $N$-((4-methoxyphenyl)sulfonyl)-3-methyl-1- $(p$ tolyl)-1H-pyrazole-4-carboxamide (3s)

Light yellow solid, yield $75 \%$; mp $89-92^{\circ} \mathrm{C}$; ${ }^{1} \mathrm{H}-\mathrm{NMR}$ $\left(600 \mathrm{MHz}, \mathrm{CDCl}_{3}\right) \delta: 8.67(\mathrm{~s}, 1 \mathrm{H}), 8.09(\mathrm{~d}, J=8.9 \mathrm{~Hz}, 2 \mathrm{H}), 7.31$ (d, $J=4.7 \mathrm{~Hz}, 3 \mathrm{H}), 7.02(\mathrm{~d}, J=8.9 \mathrm{~Hz}, 3 \mathrm{H}), 3.88(\mathrm{~s}, 3 \mathrm{H}), 2.47$ $(\mathrm{s}, 3 \mathrm{H}), 2.42(\mathrm{~s}, 3 \mathrm{H}) .{ }^{13} \mathrm{C}-\mathrm{NMR}(151 \mathrm{MHz}, \mathrm{DMSO}) \delta: 163.84$, $163.17,150.20,137.84,135.99,133.63,133.24,129.77,126.19$, 125.53, 116.87, 114.62, 55.86, 21.51, 13.71. MS (ESI): 420.8 $\left(\mathrm{C}_{19} \mathrm{H}_{18} \mathrm{ClN}_{3} \mathrm{O}_{4} \mathrm{~S},[\mathrm{M}+\mathrm{H}]^{+}\right)$. Anal. Calcd for $\mathrm{C}_{19} \mathrm{H}_{18} \mathrm{ClN}_{3} \mathrm{O}_{4} \mathrm{~S}: \mathrm{C}$, 54.35; H, 4.32; N, 10.01. Found: C, 55.28; H, 4.36; N, 10.08 .

5-Chloro-3-methyl-1-( $p$-tolyl)- $N$-((4-(trifluoromethoxy)phenyl)sulfonyl)-1H-pyrazole-4-carboxamide (3t)

Light yellow solid, yield $74 \%$; mp $129-131^{\circ} \mathrm{C} ;{ }^{1} \mathrm{H}-\mathrm{NMR}$ $\left(600 \mathrm{MHz}, \mathrm{CDCl}_{3}\right) \delta: 8.78(\mathrm{~s}, 1 \mathrm{H}), 8.22(\mathrm{~d}, J=8.7 \mathrm{~Hz}, 2 \mathrm{H}), 7.38$ (d, $J=8.4 \mathrm{~Hz}, 2 \mathrm{H}), 7.30$ (d, $J=1.6 \mathrm{~Hz}, 4 \mathrm{H}), 2.47$ (s, 3H), 2.42 (s, $3 \mathrm{H}) .{ }^{13} \mathrm{C}-\mathrm{NMR}(151 \mathrm{MHz}, \mathrm{DMSO}) \delta: 163.84,163.27,153.83$, $150.24,135.57,133.45,133.14,129.76,129.67,126.22,125.13$, 116.85, 114.65, 21.54, 13.72. MS (ESI): $474.8\left(\mathrm{C}_{19} \mathrm{H}_{15} \mathrm{ClF}_{3} \mathrm{~N}_{3} \mathrm{O}_{4} \mathrm{~S}\right.$, $\left.[\mathrm{M}+\mathrm{H}]^{+}\right)$. Anal. Calcd for $\mathrm{C}_{19} \mathrm{H}_{15} \mathrm{ClF}_{3} \mathrm{~N}_{3} \mathrm{O}_{4} \mathrm{~S}: \mathrm{C}, 48.16 ; \mathrm{H}, 3.19$; N,8.87. Found: C, 48.22; H, 3.24; N, 8.91.

In Vitro AtAHAS Inhibition The plant AtAHAS was expressed and purified as described previously. ${ }^{22)}$ AHAS activity was measured in accordance with the literature procedure. ${ }^{23)}$

In Vivo Inhibition of the Root Growth of Rape The in vivo data were determined using previously published method. ${ }^{24)}$

Molecular Docking The crystal structures of succinate dehydrogenase (PDB entry: 5k6t) were retrieved from the Protein Data Bank. The molecular docking procedure was performed by using CDOCKER protocol for receptor-ligand interactions section of DS 3.1 (Discovery Studio 3.1; Accelrys, Inc.). ${ }^{25)}$

Acknowledgments This work was supported by National Natural Science Foundation of China (Nos. 21302002 and 21602042), and by Anhui Provincial Natural Science Foundation (1408085QB33, 1508085MB33). This work has been checked by Dr. Deepak Kumar.

Conflict of Interest The authors declare no conflict of interest.

\section{References}

1) Pang S. S., Duggleby R. G., Guddat L. W., J. Mol. Biol., 317, 249262 (2002).

2) Duggleby R. G., Pang S. S., J. Biochem. Mol. Biol., 33, 1-36 (2000). 3) Chaleff R. S., Mauvais C. J., Science, 224, 1443-1445 (1984).

4) LaRossa R. A., Schloss J. V., J. Biol. Chem., 259, 8753-8757 (1984).

5) McCourt J. A., Pang S. S., King-Scott J., Guddat L. W., Duggleby R. G., Proc. Natl. Acad. Sci. U.S.A., 103, 569-573 (2006).

6) Tranel P. J., Wright T. R., Weed Sci., 50, 700-712 (2002).

7) Whaley C. M., Wilson H. P., Westwood J. H., Weed Sci., 55, 83-90 (2007)

8) Yu Q., Abdallah I., Han H., Owen M., Powles S., Planta, 230 713-723 (2009)

9) Yu Q., Han H., Powles S. B., Pest Manag. Sci., 64, 1229-1236 (2008)

10) Keter F. K., Darkwa J., Biometals, 25, 9-21 (2012).

11) Mukherjee R., Coord. Chem. Rev., 203, 151-218 (2000).

12) Dai H., Li Y.-Q., Du D., Qin X., Zhang X., Yu H.-B., Fang J.-X., J. Agric. Food Chem., 56, 10805-10810 (2008).

13) Lamberth C., Heterocycles, 71, 1467-1502 (2007)

14) Ren Z. L., Zhang J., Li H. D., Chu M. J., Zhang L. S., Yao X. K. Xia Y., Lv X. H., Cao H. Q., Chem. Pharm. Bull., 64, 1755-1762 (2016)

15) Theorell H., Yonetani T., Biochem. Z., 338, 537-553 (1963).

16) Han C., Guo Y.-C., Wang D.-D., Dai X.-J., Wu F.-J., Liu H.-F., Dai G.-F., Tao J.-C., Chin. Chem. Lett., 26, 534-538 (2015).

17) Abadi A. H., Eissa A. A. H., Hassan G. S., Chem. Pharm. Bull., 51, 838-844 (2003).

18) Ghorab M. M., Al-Said M. S., Nissan Y. M., Chem. Pharm. Bull., 60, 1019-1028 (2012)

19) Lv X.-H., Xiao J.-J., Ren Z.-L., Chu M.-J., Wang P., Meng X.-F., Li D.-D., Cao H.-Q., RSC Adv., 5, 55179-55185 (2015).

20) Garcia M. D., Nouwens A., Lonhienne T. G., Guddat L. W., Proc. Natl. Acad. Sci. U.S.A., 114, E1091-E1100 (2017).

21) Lv X.-H., Ren Z.-L., Li D.-D., Ruan B.-F., Li Q.-S., Chu M.-J., Ai C.-Y., Liu D.-H., Mo K., Cao H.-Q., Chin. Chem. Lett., 28, 377-382 (2017).

22) Chang A. K., Duggleby R. G., Biochem. J., 333, 765-777 (1998)

23) Wang J. G., Xiao Y. J., Li Y. H., Ma Y., Li Z. M., Bioorg. Med. Chem., 15, 374-380 (2007).

24) Wang J., Tan H., Li Y., Ma Y., Li Z., Guddat L. W., J. Agric. Food Chem., 59, 9892-9900 (2011).

25) Wu G., Robertson D. H., Brooks C. L., Vieth M., J. Comput. Chem. 24, 1549-1562 (2003). 